\title{
In high responding patients undergoing an initial IVF cycle, elevated estradiol on the day of hCG has no effect on live birth rate
}

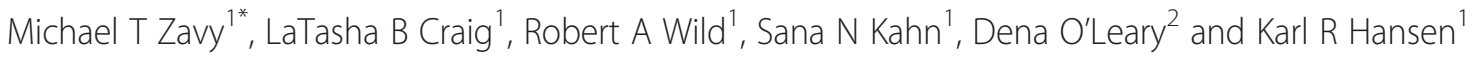

\begin{abstract}
Background: The impact of elevated estradiol on the day of human chorionic gonadotropin (hCG) administration on in vitro fertilization (IVF) outcomes has been debated for over 25 years. Some investigators have shown a positive effect, others a negative effect; while most have shown no effect. Few studies have expressed their findings based on live birth. This study examined the relationship between estradiol level and other IVF cycle response parameters in relation to pregnancy, with a focus on live births after controlling for embryo quality.

Methods: We performed a retrospective cohort study on 489 patients $<40$ years old that underwent an initial IVF cycle. Estradiol concentration on the day of hCG was categorized as; low $<2000 \mathrm{pg} / \mathrm{ml})$, mid (2001-4000 pg/ml) and high $(>4000 \mathrm{pg} / \mathrm{ml})$ to determine how estradiol level on the day of hCG affected response variables during the IVF cycle. We performed a subgroup analysis restricted to patients with good/fair quality embryos transferred $(n=428)$, to control for embryo quality and assessed pregnancy outcome. The association between estradiol and live birth (LB) was then evaluated after identifying and controlling for confounding factors. Multivariate analysis was used to identify significant main effects and interactions in the model. Estradiol levels were also compared in patients having a LB or not (NLB) in both populations.
\end{abstract}

Results: We found that estradiol was significantly related to $+\mathrm{hCG}$, clinical pregnancy rate, age, and most other IVF cycle response variables. After performing the subgroup analysis controlling for embryo quality, we found that LB rates were not different. Only the main effects of average embryo quality at transfer (AEQS), age and transferring two embryos influenced LB. Estradiol levels were also compared in patients having a LB or NLB in both populations and was found to be higher/not different in LB patients. LB rates and AEQS were also not different in a subgroup of patients having an elevated level of estradiol (>4200 pg/ml) on the day of hCG in patients having embryo transfer on day 3 or day 5.

Conclusions: After controlling for embryo quality, elevated estradiol on the day of hCG had no effect on LB.

Keywords: IVF, Estradiol level, High responder, Live birth rate, Age, Embryo quality, Day of embryo transfer

\section{Background}

In IVF cycles, high responding patients are characterized as having a large number of follicles that are associated with elevated levels of estradiol and frequently by elevated levels of progesterone on the day of hCG administration. In most situations, this results in an increased number of embryos from which candidates can

\footnotetext{
* Correspondence: michael-zavy@ouhsc.edu

'Section of Reproductive Endocrinology and Infertility Department of Obstetrics and Gynecology, Oklahoma University Health Sciences Center, Oklahoma City, Oklahoma, USA

Full list of author information is available at the end of the article
}

be chosen for embryo transfer. Due to its potential clinical importance the impact of elevated estradiol on the day of hCG on IVF outcomes has been debated for over 25 years and even after this length of the time the issue of how, or if, the supra-physiological hormonal environment exhibited in high responding patients has an adverse effect on pregnancy outcome is still not settled. Regarding elevated estradiol and pregnancy outcome, some investigators [1-4] have shown a positive effect and others [5-8] a negative effect; while most studies [9-18] have shown no effect. A meta-analysis conducted in 2004 [19] reviewed nine 
studies and concluded that no high quality evidence existed to support or refute the value of estradiol levels on the day of hCG administration as a determining factor in establishing pregnancy in IVF cycles. Possibly contributing to the disparity in outcomes from these studies are differences in the way trials were conducted, including the use of repeated measures, patient exclusion criteria, type of stimulation, day of transfer, number of embryos transferred and how estradiol levels were categorized (cutoff, percentile or area under the curve). There is also considerable variation among studies in how pregnancy rates were expressed with most using clinical pregnancy rate, with or without implantation rate. Few studies have based their conclusions on live birth outcome which arguably provides the most information on the embryos ability to tolerate the hormone environment to which it has been exposed.

The purpose of this study was to determine the relationship between the levels of estradiol on the day of hCG administration and pregnancy outcomes, with emphasis on live birth rate after controlling for embryo quality. We addressed this by initially categorizing patients according to the level of estradiol on the day of hCG administration, which has been the basis of the majority of previously published studies. We then performed additional analyses controlling for embryo quality and for confounding and interactions occurring between IVF response variables. In a secondary analysis we compared differences in estradiol levels between patients achieving a live birth and those that did not (NLB), since differences in estradiol levels have been reported in previously published reports when stratified by birth outcome $[17,20,21]$. We also tested if the day of embryo transfer made a difference in live birth (LB) rate in patients with an elevated level of estradiol on the day of hCG since some prior studies [17,20,22] had suggested that elevated estradiol only had an adverse effect on pregnancy outcome when embryo transfer was conducted on day 3.

\section{Methods}

\section{Study design}

We performed a retrospective cohort analysis (20062012) on patients that underwent an initial IVF cycle at the OU Reproductive Medicine Clinic at the University of Oklahoma. Of the 500 patients evaluated, 489 patients were included in the present study. Eleven patients were excluded due to; failed fertilization (4), only abnormal embryos available for transfer (3) or all embryos cryopreserved due to threatened ovarian hyper-stimulation (4). This investigation was approved by the Oklahoma University Health Sciences Center Institutional Review Board (\#2781, approved, 3/12/2013).

\section{Patient population and stimulation protocols}

Patients $<40$ years of age with any etiology of infertility were included in the study; no patients using donor eggs were included. Following a baseline ultrasound, all patients underwent stimulation with recombinant folliclestimulating hormone (FSH; Follistim, Organon USA or Gonal-F, Sereno) and in the majority of cases, in combination with human menopausal gonadotropin (hMG; Menopur or Repronex, Ferring Pharmaceuticals). Total units of FSH administered were calculated for each patient with the exception of 19 patients in which this information was missing. Three different protocols were utilized for patients undergoing IVF during the study interval, including long gonadotropin releasing hormone (GnRH) agonist, micro-dose flare and GnRH antagonist protocols. The particular protocol chosen was based on anticipated patient response, with the majority of patients undergoing the long agonist protocol. Follicular monitoring was carried out via serial vaginal ultrasound and serum measurement of $17 \beta$-estradiol (estradiol) and adjustments made in gonadotropin dose based upon follicular development. When 2 or more follicles were $\geq 18 \mathrm{~mm}$, patients received a subcutaneous injection of hCG (5000 or $10000 \mathrm{IU}$ ) and follicular aspiration took place 35-36 hours later. A blood sample was taken at the last ultrasound, prior to hCG administration and analyzed for estradiol. Luteal phase supplementation was by daily IM injection of progesterone $(50 \mathrm{mg})$. Fifteen days following follicle aspiration, serum hCG was measured and considered positive if $>20 \mathrm{mIU}$, biochemical pregnancy (BCP). Clinical pregnancy $(\mathrm{CP})$ was documented by observing a fetal $\operatorname{sac}(\mathrm{s})$ and heart rate by vaginal ultrasound at $6.5-7$ weeks.

\section{Hormone measurements}

Serum levels of estradiol and hCG were measured by chemiluminescent enzyme immunoassays (Immulite; Diagnostic Products, Los Angeles, CA, USA). Inter-assay and intra-assay coefficients of variation were $9.8 \%$ and $9.4 \%$ for estradiol and 5.6\% and 3.0\% for hCG.

\section{IVF-embryo transfer}

Following follicle aspiration, oocytes were held in modified human tubal fluid (mHTF) $+5 \%$ (volume/volume) human serum albumen (HSA) (In Vitro Care; IVC) until placed into individual drops of HTF $+5 \%$ HSA with oil overlay (Sage) in a humidified atmosphere of $6 \% \mathrm{CO}_{2}$ and $94 \%$ air maintained at $37^{\circ}$ until intracellular sperm injection (ICSI) or conventional IVF was carried out 4 to 6 hours following oocyte retrieval. Most cases (70\%) utilized ICSI with 25\% undergoing ICSI and conventional IVF (split) and (5\%) undergoing conventional IVF only. After injection, oocytes were placed into 50ul micro-drops of IVC-1 $+5 \%$ HSA (In Vitro 
Care) or Global medium $+10 \%$ global protein supplement (GPS; Life Global) using oil overlay and cultured in a humidified tri-gas atmosphere $\left(5-6.5 \% \mathrm{CO}_{2} ; 5 \% \mathrm{O}_{2}\right.$ and residual nitrogen) at $37^{\circ} \mathrm{C}$. Fertilization was checked between 17 and 18 hours and normally fertilized oocytes having 2 pronuclei were placed in groups of 3-4 and cultured as described previously. On day 2, embryos were graded and embryos allocated to new culture drops based on quality (good, fair or poor). A decision was made to perform embryo transfer on day 3 or day 5 , based on the number of good quality embryos. Blastocyst transfer was elected if there were at least 6 good/fair quality embryos. If this criterion was not met, there were a low number of embryos or poor quality embryos; transfer on day 3 was elected. New culture plates were made daily and embryo development and video documentation was also conducted daily at approximately 24 hour intervals.

\section{Embryo and blastocyst quality score at transfer}

Cleavage stage embryos and blastocysts were graded as being good (1), fair (2) or poor quality (3) and numerical conversion of embryo grades were performed in order to statistically analyze the data. The embryos or blastocysts that were transferred for each patient were averaged to yield an average embryo quality score (AEQS). The numeric scoring is similar to that used by the Society for Assisted Reproductive Technology [23]. For cleavage stage embryos, the quality was determined by stage of development, blastomere symmetry and fragmentation of the embryos. Our grading system uses the following designation on day 3; A (good), B (fair) C (poor) with a $(+/-)$ modifier for B quality embryos only. A good quality embryo (score of 1.0) had an A or B+ quality at the 6 cell to10 cell stage of development. A fair quality embryo (score of 2.0 for a "B" or 2.5 for a "B-") at the 5 cell to 14 cell stage of development and a poor quality (score of 3 ) if the embryo was developmentally delayed ( $\leq 4$ cells), had excessive fragmentation $>40 \%$ ) or extreme blastomere asymmetry. Blastocysts were graded as follows: a good quality blastocyst (score of 1 ) on day 5; the inner cell mass (ICM) quality was not less than $\mathrm{B}+$ quality while the trophectoderm (TE) quality was no lower than B quality. In the case of blastocysts (+/-) modifiers were used for B quality for both the ICM and TE. The minimum stage of development of good quality blastocysts was 3 (expanded) or in the case of an early observation, 2 (cavitated). A day 6 good quality blastocyst was similar to day 5 except with more advanced development. A fair quality blastocyst (score of 2) for day 5; the minimum quality was $2 \mathrm{BB}$ - and for day 6 was $4 \mathrm{BB}-$. A poor quality blastocyst (score of 3 ) for day 5 or day 6 , included any blastocyst having a B-B, or lower quality regardless of stage of development.

\section{Response variables}

We evaluated if the estradiol level on the day of hCG was associated with $\mathrm{BCP}, \mathrm{CP}$ and $\mathrm{LB}$, in patients undergoing their initial IVF cycle. Estradiol concentration on the day of hCG was categorized as; low $<2000 \mathrm{pg} / \mathrm{ml}$ ), mid (2001$4000 \mathrm{pg} / \mathrm{ml})$ and high $(>4000 \mathrm{pg} / \mathrm{ml})$ to determine how estradiol level on the day of hCG affected response variables during the IVF cycle. Given that a higher estradiol level on the day of hCG may be related to better embryo quality, we controlled for this factor by evaluating the relationship between estradiol level and outcomes in a subpopulation ( $\mathrm{n}=428$ ) containing only patients with good or fair embryo quality score at transfer. The poor quality group had an average embryo quality score range of $2.3-3.0$. To focus on LB rates, we examined if there was an association between maternal estradiol levels and LB after controlling for potential confounders using multivariate logistic regression. We examined if estradiol levels and other response variables were different by live birth status. The NLB group included those patients that did not become pregnant, or had a BCP or a CP but not a LB. We examined what effect the day of embryo transfer had on NLB and LB rate in a subgroup of patients from the entire population having an estradiol level of $>3000 \mathrm{pg} / \mathrm{ml}$ on the day of hCG. We compared LB rate, number of embryos transferred and embryo transfer quality scores in patients having embryo transfer on day 3 or day 5 .

\section{Statistical analyses}

We evaluated differences between estradiol groups for response variables using Chi-square and analysis of variance (ANOVA), considering $\mathrm{p}<0.05$ as significant. Chi-Square, Student's t-tests and univariate logistic regression was used to evaluate the association between the live births and each covariate. Multivariable logistic regression was performed, including all covariates with a significance level of $\mathrm{p} \leq 0.1$ on univariable analysis, avoiding co-linearity. The model was assessed for the presence of two way interactions and confounding, with a difference between crude and adjusted odds ratios of $>15 \%$ considered evidence of a significant confound in the regression model.

The entire population as well as the subgroup were partitioned by NLB or LB, and differences in response variables was assessed by two sample t-tests, with $\mathrm{p}<0.05$ considered significant. The same approach was taken when examining NLB and LB differences in the high estradiol populations by day of embryo transfer (day 3 or day 5). Chi square was used to evaluate differences in LB between days of transfer with a level of $\mathrm{P}<0.05$ considered significant.

\section{Results}

Patient demographics for the entire population are shown in Table 1. Pregnancy rates and IVF cycle response 
Table 1 Patient demographics of the entire population ( $n=489$ )

\begin{tabular}{lc}
\hline Parameters & Values \\
\hline Age (years) & $31.6 \pm 4.1$ \\
BMI & $25.9 \pm 6.2$ \\
FSH administered (mIU) per patient & $2969 \pm 1434$ \\
Estradiol on day of hCG (pg/ml) & $2871 \pm 1153$ \\
Oocytes retrieved per patient & $18.9 \pm 8.6$ \\
MIl oocytes per patient & $14.5 \pm 7.2$ \\
2PN embryos per patient & $9.8 \pm 5.2$ \\
Embryos transferred per patient & $2.04 \pm 0.42$ \\
Embryo quality at transfer (AEQS) per patient & $1.61 \pm 0.59$ \\
Percentage of day 3 transfers & $286(58.5 \%)$ \\
Percentage of day 5 transfers & $203(41.5 \%)$ \\
\hline
\end{tabular}

Mean \pm 1SD.

parameters for the entire population $(n=489)$ and the subgroup $(n=428)$ that had only good or fair quality embryos transferred, are summarized in Tables 2 and 3 respectively. For the entire population (Table 2), the higher estradiol groups were associated with greater biochemical and clinical pregnancy rates. Whereas, in the sub-group (Table 3), with fair to good embryo quality no association with estradiol was observed on BCP, CPR or LBR in contrast to all other IVF response variables. Although live birth rates were greatest $(\mathrm{NS}, \mathrm{P}=0.052)$ in higher estradiol groups from the categorical analysis shown in Table 2, following the univariate analysis (Table 4) in which estradiol level was evaluated as a continuous variable, estradiol level had a significant $(P=0.013)$ effect on LB as did other covariates including age, FSH dosage, number of metaphase II (MII) oocytes, total 2PN embryos, AEQS and embryo transfer day. In the multivariate analysis (Table 5) however, estradiol level was not related to live birth. Only the main effects of AEQS, patient age, and the transfer of two embryos as opposed to 1 or 3 , were found to significantly affect LB in the final model. The multivariate regression model also revealed significant two-way interactions between transfer on day 5 and patient age $(\mathrm{P}<0.01)$ and transfer on day 5 and FSH dose administered $(\mathrm{P}<0.04)$. IVF response variables in the total population and in the subgroup containing only good and fair quality embryos on the day of embryo transfer were evaluated by live birth status. In the total population for day 3 transfers (Table 6) there were differences between the NLB and LB groups in patient age, FSH dose, number of 2PN embryos and AEQS, whereas, in the subgroup-only analysis, patient age and AEQS were different. In the total population for day 5 transfers (Table 7), FSH dose, number of MII eggs and AEQS differed. In the subgroup however, only FSH dose differed between NLB and LB. In the entire population the LB rate was greater for day 5 than for day 3 transfers. However, when the subgroup containing only good and fair embryos was evaluated, although the LB rate was slightly higher $(65.3 \%$ vs $59.1 \%)$, the difference was no longer statistically significant.

In the final analysis, a subgroup of patients having embryo transfer on day 3 or day 5 that had an estradiol level of $>3000 \mathrm{pg} / \mathrm{ml}$ on the day of hCG were compared (Table 8). When embryo transfer was conducted on day

Table 2 Estradiol groups in the entire population of patients and IVF outcome

\begin{tabular}{|c|c|c|c|c|}
\hline Variables & Group $1<2000 \mathrm{pg} / \mathrm{mL}, \mathrm{n}=123$ ) & Group $2(2000-4000) \mathrm{pg} / \mathrm{ml}, \mathrm{n}=288)$ & Group $3>4000 \mathrm{pg} / \mathrm{mL}, \mathrm{n}=78$ ) & $p$-value \\
\hline Age (years) & $32.6 \pm 4.2$ & $31.5 \pm 4.1$ & $30.6 \pm 3.8$ & $<0.003$ \\
\hline BMI & $27.4 \pm 7.0$ & $26.3 \pm 6.2$ & $24.9 \pm 6.1$ & $<0.024$ \\
\hline FSH dose $(I U)^{*}$ & $3851 \pm 1454$ & $2883 \pm 1314$ & $1900 \pm 896$ & $<0.001$ \\
\hline Peak estradiol (pg/mL) & $1489 \pm 365$ & $2950.1 \pm 561$ & $4758 \pm 594$ & $<0.001$ \\
\hline Oocytes retrieved & $11.9 \pm 5.7$ & $19.9 \pm 7.5$ & $26.5 \pm 8.6$ & $<0.001$ \\
\hline MII oocytes & $8.9 \pm 4.7$ & $15.1 \pm 6.3$ & $20.7 \pm 7.3$ & $<0.001$ \\
\hline Total 2PN & $6.4 \pm 3.7$ & $10.3 \pm 4.7$ & $13.5 \pm 5.9$ & $<0.001$ \\
\hline AEQS & $1.78 \pm 0.67$ & $1.57 \pm 0.58$ & $1.48 \pm 0.44$ & $<0.001$ \\
\hline No. embryos transferred & $2.11 \pm 0.50$ & $2.04 \pm 0.40$ & $1.92 \pm 0.35$ & $<0.008$ \\
\hline BCP (\%) & $58.5^{\mathrm{a}}$ & $70.8^{b}$ & $78.2^{\mathrm{b}}$ & $<0.007$ \\
\hline CP (\%) & $49.6^{\mathrm{a}}$ & $64.2^{b}$ & $65.4^{\mathrm{b}}$ & $<0.015$ \\
\hline LB (\%) & 47.2 & 59.0 & 61.5 & 0.052 \\
\hline Total pregnancy loss (\%) & 19.4 & 16.7 & 21.3 & 0.670 \\
\hline $\begin{array}{l}\text { Poor quality embryos } \\
\text { for transfer }(\%)\end{array}$ & 19.5 & 9.7 & 1.3 & $<0.001$ \\
\hline
\end{tabular}

Mean \pm 1SD.

Chi-square and ANOVA tests as appropriate. For pregnancy related variables, within row, response variables with different superscripts differ at a minimum of $\mathrm{P}<0.05$. The number patients in the entire population were 489. *The $\mathrm{n}$ for FSH dose was 470 due to missing values. 
Table 3 Estradiol groups in a subpopulation having good or fair quality embryos for transfer and IVF outcome

\begin{tabular}{|c|c|c|c|c|}
\hline Variables & Group $1(<2000 \mathrm{pg} / \mathrm{mL}, \mathrm{n}=95)$ & Group $2(2000-4000 \mathrm{pg} / \mathrm{mL}, \mathrm{n}=257)$ & Group $3>4000 \mathrm{pg} / \mathrm{mL}, \mathrm{n}=76$ ) & $\mathrm{p}$-value \\
\hline Age (years) & $32.6 \pm 4.1$ & $31.4 \pm 3.9$ & $30.6 \pm 3.9$ & $<0.003$ \\
\hline BMl & $28.3 \pm 7.1$ & $26.2 \pm 6.1$ & $24.8 \pm 6.1$ & $<0.002$ \\
\hline FSH dose $(I U)^{*}$ & $3677 \pm 1416$ & $2834 \pm 1311$ & $1904 \pm 901$ & $<0.001$ \\
\hline Peak estradiol (pg/mL) & $1506 \pm 352$ & $2965 \pm 564$ & $4763 \pm 599$ & $<0.001$ \\
\hline Oocytes retrieved & $12.5 \pm 5.6$ & $20.2 \pm 7.4$ & $26.7 \pm 8.4$ & $<0.001$ \\
\hline Mll oocytes & $9.6 \pm 4.7$ & $15.5 \pm 6.3$ & $20.9 \pm 7.3$ & $<0.001$ \\
\hline Total 2PN & $7.0 \pm 3.5$ & $10.6 \pm 4.7$ & $13.7 \pm 5.8$ & $<0.001$ \\
\hline AEQS & $1.49 \pm 0.43$ & $1.43 \pm 0.43$ & $1.46 \pm 0.42$ & $<0.490$ \\
\hline No. embryos transferred & $2.11 \pm 0.45$ & $2.00 \pm 0.35$ & $1.91 \pm 0.33$ & $<0.002$ \\
\hline $\mathrm{BCP}(\%)$ & 71.6 & 74.3 & 78.9 & $<0.640$ \\
\hline CP (\%) & 62.1 & 68.5 & 65.8 & $<0.550$ \\
\hline LB (\%) & 58.9 & 63.0 & 61.8 & $<0.810$ \\
\hline Total pregnancy loss (\%) & 17.6 & 15.2 & 21.7 & $<0.540$ \\
\hline
\end{tabular}

Mean \pm 1SD.

Chi-square and ANOVA tests as appropriate. For pregnancy related variables, within row, response variables with different superscripts differ at a minimum of $\mathrm{P}<0.05$. The number patients in the subpopulation were 428 . ${ }^{*}$ The $n$ for FSH dose for the subpopulation was 413 due to missing values.

3 or day 5 with high quality embryos, patients had similar estradiol levels, with the number of oocytes retrieved, mature oocytes and number of 2PN embryos all being higher in the day 5 transfer group. However, there was no significant difference in the CPR or LBR for patients receiving a transfer on day 3 or day 5 .

As another part of this analysis we examined if a high level of estradiol $(>4200 \mathrm{pg} / \mathrm{ml}$ ) on the day of hCG had a deleterious effect on pregnancy rate when compared to a group having moderate estradiol levels of $>3000 \mathrm{pg} \mathrm{ml}$ and $<4200 \mathrm{pg} / \mathrm{ml}$, that had embryo transfer on day 3. As shown in Table 9, the estradiol level and number of oocytes retrieved were higher in the $>4200 \mathrm{pg} / \mathrm{ml}$ group, but there was no difference in either the CPR or LBR between groups.

\section{Discussion}

This study examined the relationship between estradiol level and other IVF cycle response parameters in relation to pregnancy, with a focus on live births after controlling for embryo quality. In high responders there is usually an increased number of eggs retrieved resulting in more embryos and therefore, better embryo selection, leading to more patients undergoing transfer on day 5 . In the case of poor responders the opposite is true as was demonstrated in the low estradiol group in Table 2. Here, there were fewer oocytes in spite of a higher dosage of $\mathrm{FSH}$, which also resulted in fewer 2PN embryos and ultimately poorer embryo selection at the time of transfer.

In our experience, patients having embryo transfer on day 3 can attain high pregnancy rates, but only if their

Table 4 Univariate analysis of the association of response variables with LB outcome

\begin{tabular}{lccr}
\hline & \multicolumn{1}{c}{ Live birth } & Yes & p value \\
\cline { 2 - 4 } & $32.26 \pm 4.30$ & $31.17 \pm 3.85$ & 0.001 \\
\hline Age & $2724 \pm 1158$ & $2984 \pm 1138$ & 0.013 \\
Estradiol level on day of hCG & $3305 \pm 1462$ & $2716 \pm 1362$ & 0.001 \\
FSH (IU administered) & $13.14 \pm 6.92$ & $15.49 \pm 7.27$ & 0.001 \\
MII oocytes & $8.83 \pm 5.13$ & $10.61 \pm 5.15$ & 0.001 \\
Total 2PN & $1.820 \pm 0.662$ & $1.448 \pm 0.476$ & 0.001 \\
AEQS & $2.075 \pm 0.499$ & $2.014 \pm 0.351$ & 0.100 \\
Number of embryos transferred & & & 0.013 \\
Embryo transfer day & $138(48.3 \%)$ & $148(51.7 \%)$ & 286 \\
Day 3 & $75(36.9 \%)$ & $128(63.1 \%)$ & 203 \\
Day 5 & 213 & 276 & 489 \\
Total & & & \\
\hline
\end{tabular}


Table 5 Multivariate logistic regression

\begin{tabular}{lcccc}
\hline Variables & Beta & Odds ratio & Confidence limit & p value \\
\hline AEQS & -1.07832 & 0.34017 & $(0.23316-0.49628)$ & 0.001 \\
Patient age & -0.16244 & 0.85007 & $(0.73570-0.98221)$ & 0.028 \\
Estradiol level & -0.00011 & 0.99989 & $0.99968-1.00010)$ & 0.308 \\
Day 5 Embryo transfer & -3.36729 & 0.03448 & $(0.00098-1.21223)$ & 0.064 \\
FSH dosage & -0.00089 & 0.99911 & $(0.99782-1.00040)$ & 0.175 \\
Two embryos transferred & 1.05610 & 2.87512 & $(1.25510-6.58623)$ & 0.013 \\
Three embryos transferred & 1.02113 & 2.77634 & $(0.91250-8.44714)$ & 0.072 \\
Day 5 transfer X Age & 0.14946 & 1.16121 & $(1.03430-1.30369)$ & 0.011 \\
Day 5 transfer X FSH dosage & -0.00034 & 0.99966 & $(0.99933-0.99999)$ & 0.042 \\
Age X FSH dosage & 0.00002 & 1.00002 & $(0.99999-1.00006)$ & 0.224 \\
\hline
\end{tabular}

embryo quality is not compromised. Therefore, we evaluated a population of patients undergoing their initial IVF cycle to determine the relationship between serum estradiol level and IVF outcomes. We next evaluated a subgroup that included patients having only good or fair quality embryos transferred to study the influence of embryo quality on pregnancy rate and subsequent live birth outcome. Although our initial categorical analysis showed that in an unselected population of first time IVF patients, estradiol level was significantly related to better pregnancy rates (Table 2), in patients having only good or fair quality embryos transferred, (to control for embryo quality influence), estradiol level was no longer related to pregnancy outcome (Table 3). Patients in the lowest estradiol group required the greatest amount of FSH, suggesting a significant component of this group was made up of poor responders. Since our primary interest was in understanding the relationship between live birth and elevated estradiol, we conducted a univariate analysis on the entire population to evaluate the association of IVF response variables with LB outcome. This analysis showed that patient age, estradiol level, $\mathrm{FSH}$ dose, number of MII oocytes, total number of $2 \mathrm{PN}$ embryos, AEQS and day of transfer (day 3 or day 5) were all related to LB rate. After multiple regression to adjust for confounders and to test for interactions, the only main effects that significantly affected LBR were, AEQS $(\mathrm{P}<0.001)$, the transfer of two embryos $(\mathrm{P}<0.01)$

Table 6 Response variables for the entire population and subgroup having good/fair quality embryos transferred on day 3

\begin{tabular}{|c|c|c|c|c|}
\hline \multirow[b]{2}{*}{ Variables } & \multicolumn{2}{|c|}{ Entire population } & \multicolumn{2}{|c|}{ Good/fair embryos only } \\
\hline & Non-LB & LB & Non-LB & LB \\
\hline Number & $138(48.3 \%)$ & $148(51.7 \%)^{*}$ & $96(40.9 \%)$ & $139(59.1 \%)^{* *}$ \\
\hline Patient age & $33.0 \pm 4.2^{a}$ & $31.4 \pm 4.0^{\mathrm{a}}$ & $32.9 \pm 3.9^{d}$ & $31.5 \pm 3.9^{d}$ \\
\hline FSH dose (IU) & $3574 \pm 1499^{b}$ & $3158 \pm 1397^{b}$ & $3423 \pm 1490$ & $3124 \pm 1381$ \\
\hline Estradiol on day of hCG pg/ml & $2451 \pm 1082$ & $2634 \pm 1061$ & $2588 \pm 1115$ & $2636 \pm 1080$ \\
\hline BMI & $26.5 \pm 6.6$ & $27.1 \pm 6.7$ & $27.1 \pm 6.4$ & $27.2 \pm 6.7$ \\
\hline Number retrieved oocytes & $15.9 \pm 8.2$ & $16.0 \pm 6.9$ & $17.2 \pm 8.6$ & $16.1 \pm 6.8$ \\
\hline Mll eggs & $11.1 \pm 6.0$ & $12.1 \pm 5.7$ & $12.0 \pm 6.2$ & $12.3 \pm 5.6$ \\
\hline Number of 2PN & $6.9 \pm 4.1^{c}$ & $8.1 \pm 3.9^{c}$ & $7.8 \pm 4.1$ & $8.1 \pm 3.8$ \\
\hline Number of embryos transferred & $2.19 \pm 0.51$ & $2.11 \pm 0.29$ & $2.17 \pm 0.43$ & $2.11 \pm 0.32$ \\
\hline AEQS & $1.91 \pm 0.68^{a}$ & $1.39 \pm 0.49^{a}$ & $1.54 \pm 0.42^{a}$ & $1.32 \pm 0.40^{a}$ \\
\hline
\end{tabular}

All values are Mean \pm 1 SD.

Differences within row comparing the same response variable by pregnancy status (NLB or LB) for the entire population or patients having only good or fair quality embryos transferred having the same superscript are different; ${ }^{a} p<0.001 ;{ }^{b} p<0.020 ;{ }^{c} p<0.010,{ }^{d} p<0.007$.

${ }^{*}$ Chi Square comparison for difference between Day 3 and Day 5 in the total population for Live Birth was $p<0.02$.

${ }^{* *}$ Chi Square comparison for difference between Day 3 and Day 5 in population with only good or fair quality embryos used for transfer for Live Birth was NS. 
Table 7 Response variables for the entire population and subgroup having good/fair quality embryos transferred on day 5

\begin{tabular}{|c|c|c|c|c|}
\hline \multirow[t]{2}{*}{ Variables } & \multicolumn{2}{|c|}{ Entire population } & \multicolumn{2}{|c|}{ Good/fair embryos only } \\
\hline & Non-LB & LB & Non-LB & LB \\
\hline Number & 75 (36.9\%) & $128(63.1 \%)^{*}$ & $67(34.7 \%)$ & $126(65.3 \%)^{* *}$ \\
\hline Patient age & $30.9 \pm 4.1$ & $30.9 \pm 3.7$ & $30.6 \pm 4.1$ & $30.9 \pm 3.7$ \\
\hline FSH dose (IU) & $2798 \pm 1250^{a}$ & $2204 \pm 1124^{a}$ & $2702 \pm 1189^{c}$ & $2203 \pm 1130^{c}$ \\
\hline Estradiol on day of hCG pg/ml & $3226 \pm 1132$ & $3390 \pm 1092$ & $3375 \pm 1088$ & $3391 \pm 1090$ \\
\hline BMI & $26.0 \pm 6.7$ & $25.5 \pm 5.9$ & $25.9 \pm 6.7$ & $25.4 \pm 5.9$ \\
\hline Number retrieved oocytes & $21.6 \pm 8.3$ & $23.9 \pm 8.3$ & $22.4 \pm 7.9$ & $23.8 \pm 8.3$ \\
\hline Mll eggs & $16.9 \pm 6.9^{b}$ & $19.4 \pm 7.0^{\mathrm{b}}$ & $17.7 \pm 6.6$ & $19.4 \pm 7.0$ \\
\hline Number of 2PN & $12.2 \pm 5.3$ & $13.5 \pm 4.9$ & $12.7 \pm 5.1$ & $13.5 \pm 4.9$ \\
\hline Number of embryos transferred & $1.87 \pm 0.41$ & $1.91 \pm 0.36$ & $1.85 \pm 0.40$ & $1.90 \pm 0.37$ \\
\hline AEQS & $1.66 \pm 0.60^{d}$ & $1.51 \pm 0.46^{d}$ & $1.51 \pm 0.42$ & $1.49 \pm 0.43$ \\
\hline
\end{tabular}

All values are Mean $\pm 1 S D$.

Differences within row comparing the same response variable by pregnancy status (NLB or LB) for the entire population or patients having only good or fair quality embryos transferred having the same superscript are different; ${ }^{a} p<0.001 ;{ }^{b} p<0.010,{ }^{c} p<0.007,{ }^{d} p<0.040$.

${ }^{*}$ Chi Square comparison for difference between Day 3 and Day 5 in the total population for Live Birth was $p<0.02$.

${ }^{*}$ Chi Square comparison for difference between Day 3 and Day 5 in populations with only good or fair quality embryos used for transfer was NS.

and the patient's age $(\mathrm{P}<0.03)$. The level of estradiol had no effect on LB rate $(\mathrm{P}=0.308)$. We also found significant two-way interactions between transfer on day 5 and patient age $(\mathrm{P}<0.01)$ as well as transfer on day 5 and FSH dose $(\mathrm{P}<0.04)$. We interpret these interactions to mean that patients achieving a LB after transfer on day 5 were younger, and also required less FSH.

Some studies [20,21] have reported that estradiol was elevated in non-pregnant patients compared to pregnant patients suggesting that an elevated estradiol level may be associated with not achieving pregnancy, although in one study [20] this was seen in patients having a day 3 transfer but not on day 5 . To examine this question, we partitioned patients on the basis of LB status by day of transfer. Results from our study (Tables 6 and 7) and another study [17] do not support this

Table 8 Comparison between patients having transfer on day 3 or day 5 with estradiol levels $>3000 \mathrm{pg} / \mathrm{ml}$ on the day of hCG with *high quality embryos transferred

\begin{tabular}{lccc}
\hline Variables & Day 3 & Day 5 & p value \\
\hline Number & 52 & 80 & \\
Estradiol level & $3859 \pm 595$ & $4037 \pm 799$ & 0.17 NS \\
Oocytes collected & $21.6 \pm 8.4$ & $26.4 \pm 8.8$ & $<0.002$ \\
Number of Mll oocytes & $15.2 \pm 6.4$ & $21.7 \pm 7.2$ & $<0.0001$ \\
Number of 2PN embryos & $8.5 \pm 4.2$ & $15.2 \pm 5.5$ & $<0.0001$ \\
Number of embryos transferred & $2.04 \pm 0.19$ & $1.85 \pm 0.36$ & $<0.006$ \\
AEQS & $1.18 \pm 0.24$ & $1.23 \pm 0.25$ & 0.25 NS \\
CPR & $33 / 52(63.5 \%)$ & $55 / 80(68.7 \%)$ & NS \\
LBR & $30 / 52(57.8 \%)$ & $52 / 80(65.0 \%)$ & NS \\
\hline
\end{tabular}

*High quality embryos were embryos having a AEQS of $1.0-1.5$. finding since estradiol was either higher or the same in LB patients compared to NLB patients.

Others $[17,20,22]$ have proposed that elevated estradiol may have a negative influence on pregnancy but only when embryo transfer is performed on day 3, arguing that a supra-physiological estradiol environment may cause embryo-endometrial asynchrony which is alleviated if transfer is delayed until day 5. To address this question, we evaluated a subset of patients that had an estradiol level of $>3000 \mathrm{pg} / \mathrm{ml}$ on the day of hCG taken from the entire population (described in Table 2), which compares IVF cycle response variables for patients that had embryo transfer on day 3 or day 5 . We chose to use the same estradiol cutoff range $(>3000 \mathrm{pg} / \mathrm{ml})$ used in a

Table 9 Comparisons between patients having day 3 embryo transfer with moderate $(>3000<4200 \mathrm{pg} / \mathrm{ml})$ or high ( $>4200 \mathrm{pg} / \mathrm{ml}$ ) estradiol levels on the day of hCG administration

\begin{tabular}{lccc}
\hline Variables & \multicolumn{2}{c}{ Estradiol Level } & p value \\
\cline { 2 - 3 } & $\begin{array}{c}\mathbf{8} \mathbf{3 0 0 0} \\
\mathbf{4} \mathbf{2 0 0} \mathbf{~} \mathbf{~ g} / \mathbf{m l}\end{array}$ & $\begin{array}{c}\mathbf{7 4 2 0 0} \\
\mathbf{~ p g} / \mathbf{m l}\end{array}$ & \\
\hline Number & 63 & 22 & \\
Estradiol level & $3529 \pm 303$ & $4872 \pm 619$ & $<0.0001$ \\
Oocytes collected & $19.24 \pm 8.12$ & $25.0 \pm 8.3$ & $<0.005$ \\
Number of 2PN embryos & $8.35 \pm 3.92$ & $10.1 \pm 4.15$ & $<0.08$ NS \\
Number of embryos & $2.19 \pm 0.40$ & $2.09 \pm 0.29$ & $<0.29$ NS \\
transferred & & & \\
AEQS & $1.62 \pm 0.66$ & $1.51 \pm 0.46$ & $<0.47$ NS \\
CPR & $37(58.7 \%)$ & $15(68.2 \%)$ & NS \\
SAB & $5(15.6 \%)$ & $3(20.0 \%)$ & NS \\
LBR & $32(50.8 \%)$ & $12(54.5 \%)$ & NS \\
\hline
\end{tabular}


recent publication [20]. Our results (Table 8) show that in contrast to this report, we found no statistical difference in CPR or LBR in patients undergoing embryo transfer on day 3 or day 5 although CPR and LBR were slightly higher for patients having transfer on day 5 . The study of Elgindy et al. [20] was a randomized controlled trial that had strict exclusion criteria (patient age $<35$ with regular cycles and a minimum of 4 good quality embryos on day 3 of culture). By design, the estradiol level, number of oocytes retrieved, number of 2PN embryos and number of good quality embryos were all similar for the day 3 and day 5 transfer groups. Although ours was a retrospective cohort study we believe it is more reflective of a typical population of patients undergoing an initial IVF cycle. In contrast to the referenced study [20] the day of embryo transfer in our study was decided based on the number and quality of embryos, an approach similar to that used in an earlier study [22]. Therefore some bias exists which placed more patients having numerous high quality embryos in the group having embryo transfer on day 5. In our study estradiol levels were not different between patients having transfer on day 3 or day 5 but there were a significantly greater number of oocytes retrieved and $2 \mathrm{PN}$ embryos with fewer embryos being transferred on day 5. Of the patients used in our study, $17 \%$ were diagnosed as having PCOS using the Rotterdam criteria [24] and therefore probably constituted a significant number of high responding patients. These differences may at least partially explain the divergent conclusions drawn from the two studies. To further examine if patients having an elevated level of estradiol on day 3 had a different pregnancy rate compared to patients having a moderate level of estradiol undergoing embryo transfer on day 3, we performed an additional subgroup analysis. Our high estradiol group had a level of $>4200 \mathrm{pg} / \mathrm{ml}$ and the moderate estradiol group had a level $>3000$ and $<4200 \mathrm{pg} / \mathrm{ml}$, which are the same ranges used by Elgindy et al. [20]. Our results, shown in Table 9, demonstrate that the average estradiol level and number of oocytes collected were higher in the high estradiol group but there was no significant difference in CPR or LBR when compared to the moderate estradiol group, which is a conclusion not supported in the previous referenced study [20]. Understanding and managing the high responding patient is a challenge to physicians in the clinical setting of IVF. There is an increased chance of patients developing ovarian hyper-stimulation syndrome (OHSS), which in our study was low, with only four patients out of 489 (0.08\%) exhibiting moderate/severe OHSS that required paracentesis, all of which were in the moderate estradiol group (2000-4000 pg/ml) described in Table 2 and none of which had any long term adverse effects. Aside from the possibility of OHSS, much has been written about supra-physiological levels of estradiol and progesterone potentially causing endometrial-embryo asynchrony which could negatively impact pregnancy rates.

Several reports have shown that in high responding patients there is a concomitant increase in estradiol, progesterone and the number of retrieved oocytes [25-27] which may have a physiological linkage. In the case of high responders, the relationship between elevated levels of estradiol and progesterone was recently examined. Wu et al. [18] found that elevated levels of estradiol were not detrimental to live birth rate when progesterone levels were $<1.05 \mathrm{ng} / \mathrm{ml}$ whereas; when progesterone levels exceeded this threshold LB rates were compromised. Another study [27] examined this same relationship except the focus was on evaluating different progesterone thresholds. Their conclusion was that high progesterone correlated with high estradiol levels and that in high responders' progesterone levels did not show any clinical impact, although a slight compromise was observed when a threshold of $1.8 \mathrm{ng} / \mathrm{ml}$ was exceeded. This study confirmed earlier work [28] which had shown that progesterone levels $>1.5 \mathrm{ng} / \mathrm{ml}$ on the day of hCG reduced the chance of pregnancy in low and normal responders but not in high responders. On the basis of our results and the results of others, high responding patients are different than poor or normally responding patients regarding pregnancy outcomes and therefore this category of patients should be evaluated aside from the general population of patients. For this reason more large scale studies need to be performed that will elucidate if high responding patients are subject to hormone thresholds that if exceeded, could impair live birth rates. A limitation of our study was that progesterone levels on the day of hCG were not included, but unfortunately these values were not available. It is also important to point out that it is generally accepted that the supra-physiological hormone environment found in high responding patients does not have a negative effect on embryo quality or developmental potential. This has been borne out by studies in which embryos exposed to this environment and transferred to embryo recipients [11] or cryopreserved and transferred in a subsequent cycle [6] had no adverse effect on pregnancy rates. Results from our study indicate that, at least in the case of elevated levels of estradiol, no adverse effect on live birth rate is seen, regardless if embryo transfer was conducted on day 3 or day 5 . Rather, our results show that the quality of transferred embryos and patient age are the primary determinants of having a live birth.

\section{Conclusions}

We believe that our study has demonstrated that after controlling for embryo quality, an elevated level of 
estradiol on the day of hCG is neither directly detrimental nor advantageous to having a live birth. Our study shows that average embryo quality at transfer is the primary determinant in establishing a LB while patient age and the transfer of two embryos are also important. Significant interactions showed that there is interplay occurring between embryo transfer on day 5 and a lower dose requirement of FSH and younger patient age, both of which appear to contribute to an increased LB rate. An elevated level of estradiol should be viewed in the context of being part of the profile of a "high responder" requiring less FSH, more oocytes retrieved, more mature oocytes and more 2PN embryos. Yet, it is interesting that all of these response variables were found to not impact LB rate. On the basis of comparable live birth rates, our data also suggest that embryos of high quality appear to tolerate an arguably sub-optimal uterine environment that has been exposed to supra-physiological levels of estradiol.

While our study has demonstrated that elevated estradiol has no effect on live birth rate, in the light of some recent studies [29-31] showing that elevated estradiol may interfere with some aspects of placentation which in turn can contribute to an increased incidence of preeclampsia and small for gestational age (SGA) offspring, it is imperative that larger prospective studies be conducted to determine if this hormone environment is responsible for these effects. If the initial findings are substantiated perhaps it is time to seek a more comprehensive definition of IVF success that encompasses fetal and neonatal well-being and to look beyond live birth as the singular metric by which IVF success is defined.

\section{Abbreviations}

2PN: Embryos with two pronuclei; ANOVA: Analysis of variance; AEQS: Average embryo quality score; BCP: Biochemical pregnancy; CP: Clinical pregnancy; CPR: Clinical pregnancy rate; FSH: Follicle stimulating hormone; GnRH: Gonadotropin releasing hormone; GPS: Global protein supplement; hCG: Human choronic gonadotropin; hMG: Human menopausal gonadotropin; HSA: Human serum albumen; HTF: Human tubal fluid; ICM: Inner cell mass; ICSI: Intracellular sperm injection; IM: Intramuscular; IVF: In Vitro Fertilization; LB: Live birth; LBR: Live birth rate; MII: Metaphase II; mHTF: Modified (HEPES) human tubal fluid; NLB: Non-live birth; TE: Trophectoderm.

\section{Competing interests}

The authors declare that they have no competing interests.

\section{Authors' contributions}

$\mathrm{MZ}$ and $\mathrm{KH}$ played a role in the conception and design, analysis and interpretation of the data and drafting of the manuscript. LC played a role in the conception, design and review of the manuscript, RW played a role in the analysis and interpretation of the data and review of the manuscript. SK played a role in the initial conception and design of the manuscript and DO'L aided in the statistical interpretation of the data. All authors read and approved the final manuscript.

\section{Acknowledgements}

The authors thank Mr. Michael Bradford Zavy (Section of Reproductive Endocrinology and Infertility) for his help in database management used in this study.

\section{Author details}

${ }^{1}$ Section of Reproductive Endocrinology and Infertility Department of Obstetrics and Gynecology, Oklahoma University Health Sciences Center, Oklahoma City, Oklahoma, USA. ${ }^{2}$ Section of Uro-gynecology, Department of Obstetrics and Gynecology, Oklahoma University Health Sciences Center, Oklahoma City, Oklahoma, USA.

Received: 10 September 2014 Accepted: 20 November 2014 Published: 28 November 2014

\section{References}

1. Chenette PE, Sauer MV, Paulson RJ: Very high serum $E_{2}$ levels are not detrimental to clinical outcome of in vitro fertilization. Fertil Steril 1990, 54:858-863.

2. Gelety TJ, Buyalos RP: The influence of supraphysiologic $E_{2}$ levels on human nidation. J Assist Reprod Genet 1995, 12:406-412.

3. Blazar AS, Hogan JW, Frankfurter D, Hackett R, Keefe DL: Serum estradiol positively predicts outcomes in patients undergoing in vitro fertilization. Fertil Steril 2004, 81:1707-1709.

4. Kummer N, Benadiva C, Feinn R, Mann J, Nulsen J, Engmann: Factors that predict the probability of a successful clinical outcome after induction of oocyte maturation with a gonadotropin-releasing hormone agonist. Fertil Steril 2011, 96:63-68.

5. Simon C, Cano F, Valbuena D, Remohi J, Pellicer A: Clinical evidence for a detrimental effect on uterine receptivity of high serum oestradiol concentrations in high and normal responder patients. Hum Reprod 1995, 10:2432-2437

6. Ng EHY, Yeung WSB, Lau EYL, So WWK, Ho PC: High serum estradiol concentrations in fresh IVF cycles do not impair implantation and pregnancy rates in subsequent frozen- thawed embryo transfer cycles. Hum Reprod 2000, 15:250-255.

7. Chen QJ, Sun XX, Li L, Gao XH, Wu Y, Gemzell-Danielsson K, Chen LN: Effects of ovarian high response on implantation and pregnancy outcome during controlled ovarian hyperstimulation (with GnRH agonist and rFSH). Acta Obstet Gynecol Scand 2007, 86:849-854.

8. Arslan M, Bocca S, Ozturk E, Hakan A, Duran E, Stadtmauer L, Oehninger S: Cumulative exposure to high estradiol levels during the follicular phase of IVF cycles negatively affects implantation. J Assist Reprod Genet 2007, 24:111-117.

9. Sharara Fl, McClamrock HD: High estradiol levels and high oocyte yield are not detrimental to in vitro fertilization outcome. Fertil Steril 1999, 72:401-405

10. Levi AJ, Drews MR, Bergh PA, Miller BT, Scott RT: Controlled ovarian hyperstimulation does not adversely affect endometrial receptivity in in vitro fertilization cycles. Fertil Steril 2001, 76:670-674.

11. Pena JE, Chang PL, Chan LK, Zeitoun K, Thornton MH, Sauer MV: Supraphysiological estradiol levels do not affect oocyte and embryo quality in oocyte donation cycles. Hum Reprod 2002, 17:83-87.

12. Papageorgiou T, Guibert J, Goffinet F, Patrat C, Fulla Y, Janssens Y, Zorn JR: Percentile curves of serum estradiol levels during controlled ovarian stimulation in 905 cycles stimulated with recombinant FSH show that high estradiol is not detrimental to IVF outcome. Hum Reprod 2002, $17: 2846-2850$

13. Ulug U, Tosun S, Jozwiak EA, Mesut A, Sismanohlu A, Bahceci M: Subclinical pregnancy losses among women undergoing in-vitro fertilization with ICSI. J Assist Reprod Genet 2006, 23:261-267.

14. Wu CH, Kuo TC, Wu HH, Yeh GP, Tsai HD: High serum estradiol levels are not detrimental to in vitro fertilization outcome. Taiwan J Obstet Gynecol 2007, 46:54-59.

15. Kong GWS, Cheung LP, Haines CJ: Comprehensive assessment of serum estradiol impact on selected physiologic markers observed during invitro fertilization and embryo transfer cycles. J Exp Clin Assist Reprod 2009, 6:5-13.

16. Yoldemir T, Fraser IS: The effect of elevated serum estradiol levels on the day of human chorionic gonadotropin injection on pregnancy outcomes in an assisted reproduction program. Aust N Z J Obstet Gynecol 2009, 49:545-550.

17. Kyrou D, Popovic-Todorovic B, Fatemi HM, Bourgain C, Haentjens P, Van Landuyt L, Devroey P: Does the estradiol level on the day of human chorionic gonadotrophin administration have an impact on pregnancy 
rates in patients treated with rec-FSH/GnRH antagonist? Hum Reprod 2009, 24:2902-2909.

18. Wu Z, Li R, Ma Y, Deng B, Zhang X, Meng Y, Chen X, Liu P, Qiao J:

Effect of HCG-day serum progesterone and oestradiol concentrations on pregnancy outcomes in GnRH agonist cycles. Reprod BioMed Online 2012, 24:511-520.

19. Kosmas IP, Kolibianakis EM, Devroey P: Association of estradiol levels on the day of hCG administration and pregnancy achievement in IVF: a systematic review. Hum Reprod 2004, 19:2446-2453.

20. Elgindy EA, Abou-Setta AM, Mostafa MI: Blastocyst-stage versus cleavagestage embryo transfer in women with high oestradiol concentrations; randomized controlled trial. Reprod BioMed Online 2011, 23:789-798.

21. Moraloglu O, Tongue EA, Ozel M, Ozaksit G, Var T, Sarikaya E: The effect of peak and mid-luteal estradiol levels on in vitro fertilization outcome. Arch Gynecol Obstet 2012, 285:857-862.

22. Chen CH, Zhang X, Barnes R, Confino E, Milad M, Puscheck P, Kazer RR: Relationship between peak serum estradiol and treatment outcome in in vitro fertilization cycles after embryo transfer on day 3 or day 5 . Fertil Steril 2003, 80:75-79.

23. Racowsky C, Vernon MV, Mayer J, Ball GD, Behr B, Pomeroy KO, Wininger D, Gibbons W, Conaghan J, Stern JE: Standardization of grading embryo morphology. Fertil Steril 2010, 94:1152-1153.

24. Fr DD, Tarlatzis R: Revised 2003 consensus on diagnostic criteria and long-term health risks related to polycystic ovarian syndrome. Fertil Steril 2004, 81:19-25.

25. Kyrou D, AlAzemi M, Papanikolaou EG, Donoso P, Tziomalos K, Devroey P, Fatemi HM: The relationship between premature progesterone rise with serum estradiol levels and number of follicles in GnRH antagonist/ recombinant FSH-stimulated cycles. Eur J Obstet Gynecol Reprod Biol 2012, 162:165-168.

26. Fatemi HM, Doody K, Griesinger G, Witjes H, Mannaerts B: High ovarian response does not jeopardize ongoing pregnancy rates and increases cumulative pregnancy rates in $\mathrm{GnRH}$-antagonist protocol. Hum Reprod 2013, 28:442-452.

27. Requena A, Cruz M, Bosch E, Meseguer M, Garcia-Velasco JA: High progesterone levels in women with high ovarian response do not affect clinical outcomes: a retrospective cohort study. Reprod Biol and Endocrinol 2014, 12:69-75.

28. Griesinger G, Mannaerts B, Andersen CY, Witjes H, Kolibianakis EM, Gordon $\mathrm{K}$ : Progesterone elevation does not compromise pregnancy rates in high responders: a pooled analysis of in vitro fertilization patients treated with recombinant follicle-stimulating hormone/gonadotropin-releasing hormone antagonist in six trials. Fertil Steril 2013, 100:1622-1628.

29. Farhi J, Haroush AB, Andrawus N, Pinkas H, Sapir O, Fisch B, Ashkenazi J: High serum oestradiol concentrations in IVF cycles increase the risk of pregnancy complications related to abnormal placentation Reprod BioMed Online 2010, 21:331-337.

30. Imudia AN, Awonuga AO, Doyle JO, Kaimal AJ, Wright DL, Toth TL, Styer AK: Peak serum estradiol level during controlled ovarian hyperstimulation is associated with increased risk of small for gestational age and preeclampsia in singleton pregnancies after in vitro fertilization. Fertil Steril 2012, 97:1374-1379.

31. Hu XL, Feng C, Lin XH, Zhong ZX, Zhu YM, Lv PP, Lv M, Meng Y, Zhang D, Lu XE, Jin F, Sheng JZ, Xu J, Huang HF: High maternal serum estradiol environment in the first trimester is associated with the increased risk of small-for-gestational-age birth. J Clin Endocrinol Metab 2014, 99:2217-2224.

doi:10.1186/1477-7827-12-119

Cite this article as: Zavy et al:: In high responding patients undergoing an initial IVF cycle, elevated estradiol on the day of hCG has no effect on live birth rate. Reproductive Biology and Endocrinology 2014 12:119.

\section{Submit your next manuscript to BioMed Central and take full advantage of:}

- Convenient online submission

- Thorough peer review

- No space constraints or color figure charges

- Immediate publication on acceptance

- Inclusion in PubMed, CAS, Scopus and Google Scholar

- Research which is freely available for redistribution

Submit your manuscript at www.biomedcentral.com/submit
Ciomed Central 